\title{
Severe Idiopathic Gingival Fibromatosis and Electrosurgical Management: Case Report
}

\author{
Hanan Oubenyahya and Mohammed Kamal Fiqhi
}

\section{ABSTRACT}

\begin{abstract}
Idiopathic gingival fibromatosis is a rare benign oral disorder characterized by non-hemorrhagic, gradually progressive fibrous gingival enlargement. This overgrowth can be so severe that it can impact both maxillaries, on lingual and buccal sides, sometimes completely covering the crowns. This condition can begin during primary dentition and continue throughout adulthood, setting up a cascade of stomatognathic and psychological negative effects. Herewith, we report a case of a non-syndromic 14 year old female who presented with generalized severe gingival enlargement involving both arches and subsequently diagnosed as idiopathic gingival fibromatosis. The chief complaints were unaesthetic appearance and lack of eruption. The excess tissue was surgically removed under general anesthesia, using electrosugery. The patient was issued regular follow-ups to ensure proper oral hygiene and periodontal controls. Awareness of this condition is of utmost importance for early diagnosis and to prevent further complications spilling into adult age.
\end{abstract}

Keywords: gingival overgrowth, gingival fibromatosis, gingivectomy.
Published Online: April 30, 2021

ISSN: $2684-4443$

DOI: $10.24018 /$ ejdent.2021.2.2.49

Hanan Oubenyahya *

Pediatric Dentistry Department, Military Hospital, Agadir, Morocco.

(e-mail: hanan.kat ${ }^{@}$ hotmail.fr)

Mohammed Kamal Fiqhi

Maxillofacial Surgery Department, Military Hospital, Agadir, Morocco.

(email: fiqhimohammedkamal@gmail.com)

*Corresponding Author

\section{CASE REPORT}

Most cases of gingival enlargement can be attributed to bacterial plaque and poor hygiene. However, this overgrowth can also be drug induced (anticonvulsants, calcium channel blockers, immunosuppressive agents), or associated with puberty, pregnancy, diabetes, or systemic diseases. In some rare cases, it can be of genetic origins.

Gingival fibromatosis (GF) refers to a set of genetically heterogeneous conditions characterized by the proliferative enlargement of the gingiva caused by an increase in the size of submucosal collagenous connective tissue. GF can be either hereditary or idiopathic [1].

Idiopathic GF (IGF) is mainly a diagnosis by exclusion. It should be made after investigations established lack of family history and ruled out other etiologies such as the presence of systemic conditions, medical intake, or hormonal imbalance [2].

Gingival tissues are usually of normal coloration, nonbleeding with a firm fibrotic consistency and abundant stippling. The hyperplasic tissue can be nodular and localized or, the most common form, symmetrical and uniform. The excess growth is usually painless and does not extend to alveolar mucosa or bone. In its most severe forms, it can involve lingual and buccal sides of both maxillaries, partially or completely covering the crowns. This can lead to a plethora of problems: malocclusion, speech and mastication impediments, abnormal swallowing pattern, restricted lingual movement, difficulty in maintaining good oral hygiene, diastemas, delayed tooth eruption, bi-maxillary protrusion, and unaesthetic appearance [3], [4].
A 14 year old female, accompanied by her caregiver reported to our department complaining of facial disfigurement and impacted teeth due to generalized swelling of the gums. The patient gave a history of increased gum size since primary dentition. She reported being operated on two times in the past, once at the age of 3 years old and again at the age of 7 years old. Both interventions focused on locally uncovering some of the non-erupted teeth and gave way to recurrences.

The patient had a symmetrical face with incompetent lips and protruding maxillaries. Her physical appearance and psychomotor skills were normal.

Intraoral examination showed impressive, generalized enlargement of gingiva in both mandibular and maxillary arches covering almost all crowns until the premolar region. Pinkish pale with some melanin pigmentation, the gingiva was firm and not susceptible to bleeding upon probing. A Vshaped palate and huge diastema between superior incisives were also noted (Fig. 1).

The patient was referred for an orthopantomogram which showed no bone loss, a retained upper left second premolar and upper right and left distomolars (Fig. 2).

Laboratory investigations included complete blood count, thyroid and parathyroid tests, calcium, and alkaline phosphatase levels. They all turned within normal limits.

Medical and family history was noncontributory. The patient denied taking any medications that could explain the gingival enlargement.

Based on these findings a provisional diagnosis of IGF was made. 


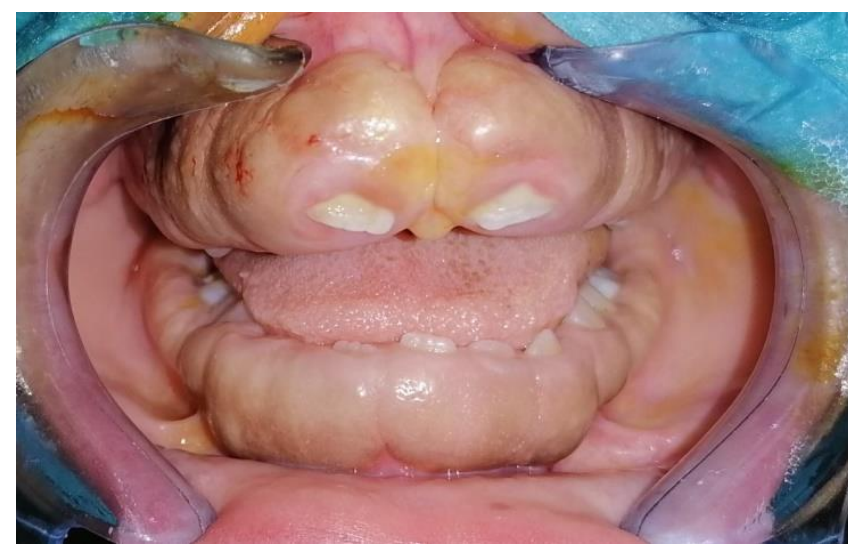

Fig. 1. Pre-operative frontal view showing generalized gingival overgrowth.

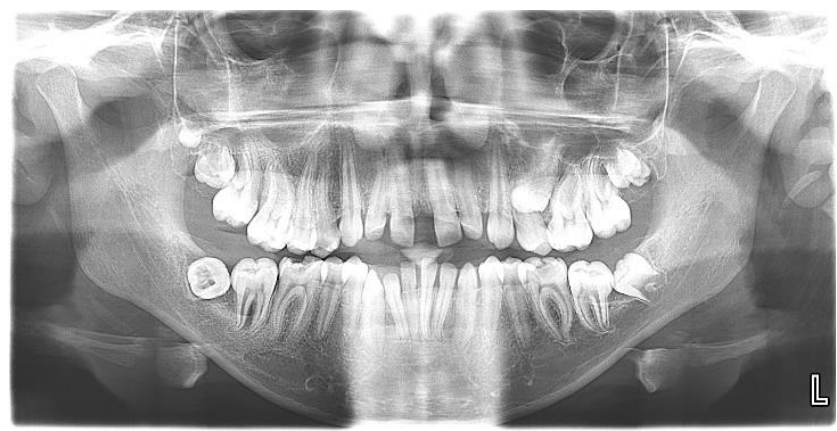

Fig. 2. Panoramic radiograph showing the presence of a retained upper left second premolar, and upper right and left distomolars.

A biopsy was performed pre-surgery. Histopathology showed an overlying hyperplasic epithelium with discrete parakeratotic hyperkeratosis and enlarged rete ridges. The cells were devoid of any cytonuclear atypia and between them was noted the proliferation of an essentially lymphoplasmacytic inflammatory infiltrate. The chorion was the site of a dense fibrosis. Malignancy signs were also ruled out. All these features led to the final diagnosis of idiopathic gingival fibromatosis (Fig. 3).

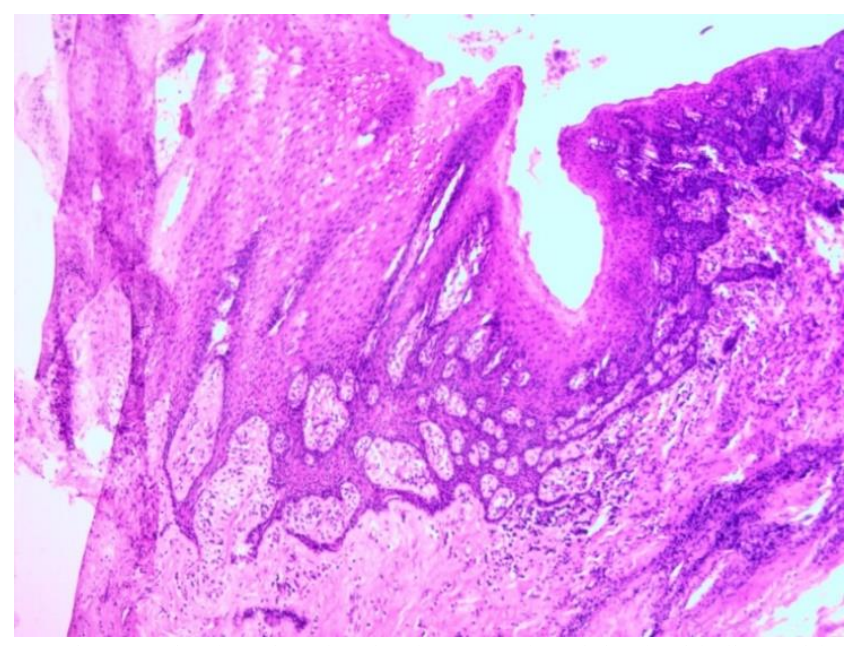

Fig. 3. Stained section showing hyperparakeratinized epithelium with dense fibro collagenous tissue and areas of chronic inflammatory cell infiltrate ( $\mathrm{H}$ and $\mathrm{E}$, medium power magnification)

Following routine phase I periodontal therapy, the treatment we opted for was electrosurgical gingivectomy of both maxillaries under general anesthesia. The procedure was thoroughly explained to the patient and caregiver and consent was obtained. In each of the four quadrants, external bevel incision was performed using a disposable hand controlled electrosurgical pencil while looking to respect gingival architectural contours. After removal of excess tissues in bulk, a thorough irrigation was done. In some sites, extensive curettage was needed, and sutures were performed. A periodontal dressing was placed to protect the exposed wound, promote healing, and reduce discomfort. The patient was put under antibiotics and analgesic medication and postoperative instructions were reinforced. Favorable healing was seen post-operatively at one week and dressing was removed. A $0.2 \%$ chlorhexidine rinse after brushing twice a day for two weeks to reduce plaque formation was prescribed. A recall schedule was set up every week for 6 weeks for maintenance. The patient was satisfied with the results and was able to keep a good level of oral hygiene (Fig. 4). However, due to the long period in time during which the patient was used to an atypical tongue positioning and a very narrow palate, she was found to be in need of speech exercises post the extensive tissue excision. Rehabilitative exercises were scheduled biweekly with a speech language pathologist for 6 weeks.

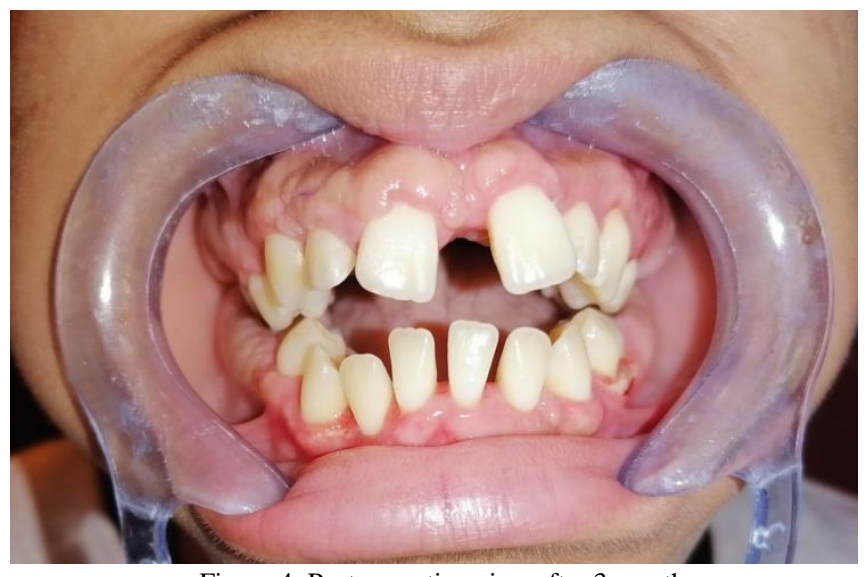

Figure 4: Post-operative view after 3 months.

\section{DISCUSSION}

This paper reports a case of IGF dating back to primary dentition. Gingival hyperplasia in children is mostly linked in the following order to: drug intake, hereditary factors, neurofibromatosis, leukemia or associated to various syndromes [5].

A proper differential diagnosis is critical before rendering an IGF diagnosis. In our case, the patient was non-syndromic and reported no distinct family or medical history, with biological investigations devoid of any abnormalities.

The exact pathogenesis of IGF is still unknown; however, it seems to be linked to a genetic predisposition [6]. The initial gingival growth, and subsequent recurrence reported in the literature seem to be associated to the presence of teeth [2], [7]-[9].

It is mainly confined to the attached gingiva and papillae, which means that periodontitis and bone involvement in such cases is the result of secondary inflammation due to plaque accumulation. It has been reported in the literature that the best time to intervene is when all the permanent teeth have erupted [10].

Various procedures are available for removal of excess gingival tissue growth, the most effective one when there is no attachment and bone loss is external bevel gingivectomy 
[11]. However, since this method usually leaves a large exposed bevel that might result in some post-operative discomfort, the choice was made to use electrosurgery. The latter was also preferred to lasers because of the fibrous density that might make it difficult to cut through with a laser. This has allowed for better hemostasis and a better definition of gingival contours to facilitate oral hygiene [12]. The choice of general anesthesia was made due to the full mouth intervention. A series of appointments was deemed too uncomfortable for the patient and the risk of prolonged soreness after each appointment was of concern. During the intervention, a foot down position of the operating table allowed for better visibility of the mandible. Local adrenaline injection and a tongue swab with surgical suction granted increased hemorrhage control.

Post operatively, literature reports conflicting recurrence rates with some linked to poor plaque control [3], [13] and others to unsatisfactory contour of marginal gingiva postsurgery [10]. The risk of recurrence and its unpredictability can have a psychological impact on the patient, especially the younger ones [14]. However, the surgery benefits in terms of aesthetics, phonetics and function far outweigh that risk, and the maintenance of good oral hygiene is of utmost importance to consolidate the surgery results. Regular follow up appointments were planned to ensure proper monitoring of the periodontal condition, caries control as well as to spot any recurrence signs.

An interesting finding from this case is the evolution of speech pattern before and after the intervention. The relationship between speech alteration and gingival procedures has received limited attention in the literature. Tongue palate contact plays a major role in pronunciation and any alteration in palato gingival anatomy can lead to speech disruption [15]. Subjective evaluation was done with visual analogue scale (VAS) score (Good pronunciation - 0, poor pronunciation - 10) to analyze the degree of speech disturbance before the surgery. The VAS score was back then evaluated at 5. Right after the excision of excess palatal tissue, a period of speech unintelligibility occurred that necessitated a 6 week therapy with a speech pathologist. Significant improvement was observed especially in regards to enunciation of the sounds " $t$ ", " $d$ ", " $n$ ", "l" and "th".

The patient was also advised to undergo orthodontic treatment later to treat her open bite and misaligned teeth. As there is no standard protocol for such treatment on patients with gingival fibromatosis, and the risk of recurrence being always present, special emphasis was put on a tight collaboration between practitioners in case the patient chooses to pursue orthodontic management.

\section{CONCLUSION}

This case highlights a non-syndromic gingival fibromatosis and its management. Dental practitioners should be aware of this condition for an appropriate early diagnosis. The latter would prevent a spillover of oral complications into adulthood and improve the patient's quality of life while growing up.

\section{REFERENCES}

[1] Shetty A, Gupta N, Shetty D, et al. Idiopathic gingival enlargement associated with generalized aggressive periodontitis in a 19-year-old female. J Indian Soc Periodontol. 2014; 18: 244-248.

[2] Ko YC, Farr JB, Yoon A, et al. Idiopathic gingival fibromatosis: case report and review of the literature. Am J Dermatopathol 2016; 38: e6871.

[3] Baptista IP. Hereditary gingival fibromatosis: A case report. J Clin Periodontol 2002; 29: 871-4.

[4] Bittencourt LP, Campos V, Moliterno LF, Ribeiro DP, Sampaio RK. Hereditary gingival fibromatosis: review of the literature and a case report. Quintessence Int. 2000 Jun; 31(6): 415-8.

[5] Rahul M, Gauba K, Gorwade N, et al. Rare case report of idiopathic gingival fibromatosis in childhood and its management. BMJ Case Rep 2019; 12: e227942.

[6] Lobo TM, Pol DG, Pol SD. Idiopathic gingival fibromatosis with asymmetrical presentation and electrosurgical management. J Indian Soc Periodontol. 2016;20(1): 98-102.

[7] Tiwana PS, De Kok IJ, Stoker DS, Cooper LF. Facial distortion secondary to idiopathic gingival hyperplasia: surgical management and oral reconstruction with endosseous implants. Oral Surg Oral Med Oral Pathol Oral Radiol Endod. 2005;100(2): 153-157.

[8] Gupta N, Maheshwari S. Advanced gingival fibromatosis. J Indian Dent Assoc. 1996; 167: 46-7.

[9] Jaju PP, Desai A, Desai RS, Jaju SP. Idiopathic gingival fibromatosis: case report and its management. Int J Dent, 2009, 2009: 153603.

[10] Emerson TG. Hereditary gingival hyperplasia. A family pedigree of four generations. Oral Surg Oral Med Oral Pathol. 1965; 19: 1-9.

[11] Ramer M, Marrone J, Stahl B, Burakoff R. Hereditary gingivalfibromatosis; Identification, treatment, control. J Am Dent Assoc. 1996; 127: 493-95.

[12] Kang CM, Lee JH, Choi HJ, Song JS, Kim SO. Long-term Management of a Gingival Fibromatosis Patient with the Primary Dentition. J Korean Acad Pediatr Dent 2014; 41(4): 328-334.

[13] Ramer M, Marrone J, Stahl B, Burakoff R. Hereditary gingival fibromatosis: identification, treatment, controlJ Am Dent Assoc. 1996 Apr; 127(4):493-5.

[14] Devi PK, Kumar GP, Bai YD, Ammaji AD. Ipsilateral idiopathic gingival enlargement and it's management using conventional gingivectomy and diode laser: A recurrent case after 15 years. J. Indian Soc. Periodontol., 17(3): 387-90, 2013.

[15] Balasundaram A, Vinayagavel M, Bandi DP. Speech intelligibility after gingivectomy of excess palatal tissue. Contemp Clin Dent. 2014 Jul; 5(3): 406-9.

\section{Oubenyahya Hanan}

Educational background

DDM, Faculty of dental medicine, Rabat, Morocco (2010);

Postgraduate Diploma, Statistical Methods in Healthcare, Bordeaux, France (2011);

CESU, Fixed Prosthodontics, Marseille, France (2014) ;

CESU, Gnathology, Marseille, France (2015) ;

Postgraduate Diploma, Pediatric Dentistry, Casablanca, Morocco (2018).

She has steadily worked at the Dental department in the Military Hospital in Agadir since 2010, setting a Pediatric Dental unit since 2018. She has also worked for a year (2020) as a UN dentist in the Democratic Republic of the Congo. 